Kamal, N.H.N. and F.A. Manan, Photosynthetic-Related Properties of Oil Palm Leaves Treated with Different Amount of Fertilizer. International Journal of Life Sciences and Biotechnology, 2020. 3(1): p. 70-80. DOI: $10.38001 /$ ijlsb.697738

\title{
Photosynthetic-Related Properties of Oil Palm Leaves Treated with Different Amount of Fertilizer
}

\author{
Nur Hanis Nor Kamal ${ }^{1}$ (D) Fazilah Abd Manan' $^{1 *}$
}

\begin{abstract}
Oil palm trees need suitable amount of fertilizer for improvement of growth and productivity. Different oil palm planting materials would require different amount of nitrogen-potassium (NK) fertilizer. Nitrogen promotes growth of plant leaves while potassium helps overall function of oil palm including formation of sugar and starch. This study focused on the determination of photosynthetic-related properties including the amount of soluble sugar, starch and chlorophyll from six planting materials of oil palm indicated as A,B,C,D,E and F. Palms were supplemented with different levels of fertilizer; T1 for control, T2 and T3. In this study, we observed that the application of the fertilizer at T2 and T3 levels for planting materials $\mathrm{A}, \mathrm{B}$, and $\mathrm{C}$ resulted in the increased amount of total soluble sugar compared to control, T1. The total starch content and the total chlorophyll content fluctuated between treatments and types of planting materials, hence no clear trend was observed. These results could be used to estimate the behaviour or biochemical responses of oil palm from different planting materials towards different levels of NK fertilizer.
\end{abstract}

\author{
ARTICLE HISTORY \\ Received \\ 6 March 2020 \\ Accepted \\ 27 March 2020 \\ KEYWORDS: \\ Oil palm, \\ planting materials, \\ fertilizer
}

\section{Introduction}

Oil palm is one of the main commodity in Malaysia with over 19.5 million tonnes of crude palm oil produced from a planted area of 5.8 million hectares in 2018. The management of this huge industry requires holistic integration in the aspect of socioeconomic and environmental sustainability [1]. To cater the needs of environmental protection and at the same time achieving high productivity in oil palm growth and yield production, effective fertilizer management is crucial. Suitable type and amount of fertilizer will help to boost the growth of plants besides preventing plant toxicity and pollution problems [2]. Nutrient deficiency in oil palm can be seen through the leaf color and other plant growth morphology. Nitrogen is one of the main macronutrients, and naturally limiting element for plant growth. Nitrogen fertilizer is important for growth of

\footnotetext{
${ }^{1}$ Department of Biosciences, Faculty of Science, Universiti Teknologi Malaysia, 81310 Skudai, Johor, Malaysia.

*Corresponding Autor: Fazilah Abd Manan, e-mail: m-fazilah@utm.my
} 
oil palm, promotes green color and helps to avoid leaf yellowing. As the main component in amino acids synthesis, application of fertilizer containing nitrogen will help plant development, hence increase crop yields $[3,4]$.

Nitrogen can improve the yield of oil palm by $49 \%$ in the presence of high potassium rate, similar as $25 \%$ yield of oil palm can be gained in response to potassium when high nitrogen rate was applied [5]. The fertilizer also enhances photosynthesis and photoprotection of plant, which help in high yield production. However, excessive amount of nitrogen, especially nitrate, gives rise to nitrogen pollution of soils and underground waters [6]. Nitrogen over-fertilization will also cause certain nutrients deficiencies, which lead to a reduction in plant growth, crop quality, and potentially lead to nitrogen loss due to soil erosion, leaching below the root zone rather than being absorbed effectively by the plant [6]. Besides nitrogen, potassium is another element that is important for oil palm functions, including the formation of sugar and starch. Hence, a recommended fertilizer level for optimum growth of oil palm could be referred in operating the plantation [7]. For planters, quality of seeds is also important to ensure good yield production. Hence, choosing the right planting material from the begining is crucial. Different progenies might have different requirements for nutrients, dissimilar efficiencies in nutrient uptake and utilization of resources [8]. Therefore, selection of high yielding planting materials with sustainable and affordable fertilization is preferred. To date, the optimum amount of NK fertilizer required vary greatly among planting materials.

Therefore, this study mainly focused on the effect of different amount of fertilizer on oil palm photosynthetic-related properties of the leaves including soluble sugar, starch, and chlorophyll content

\section{Methodology}

\section{Plant Samples}

Leaves samples from four-year old oil palm planting materials, designated as A, B, C, D, $\mathrm{E}$ and F, were collected from an oil palm plantation in Johor, Malaysia. These plants were supplied with different level of NK fertilizer indicated with T1 as control, T2 as the first level amount of NK and T3 as the second level of fertilizer (doubled amount of NK in $\mathrm{T} 2)$. 


\section{Determination of soluble sugar content}

Mixture of $0.5 \mathrm{~g}$ leaf samples and distilled water was made in $15 \mathrm{ml}$ conical tube and the volume was made up to $10 \mathrm{ml}$ with distilled water. The mixture was vortexed and incubated for 10 minutes. Anthrone, $0.2 \mathrm{~g}$ was dissolved in $100 \mathrm{ml}$ of $95 \%$ sulphuric acid. Standard curve was obtained by using sucrose as the standard solution for sucrose quantification. The centrifugation of ground dry sample and distilled water was carried out for 10 minutes at $3400 \mathrm{rpm}$. The supernatant was gained by performing a filtration. The mixture of $4 \mathrm{ml}$ sample and $8 \mathrm{ml}$ anthrone reagent was incubated for 5 minute in a water bath at $100^{\circ} \mathrm{C} \cdot \mathrm{An}$ absorbance reading was obtained by a spectrophotometer (UV160U) at wavelength of $620 \mathrm{~nm}$. The results were reported in mg sucrose per gram dry sample using sucrose standard curve plotted before [9].

\section{Determination of starch content}

The homogenization of $0.5 \mathrm{~g}$ dry leaf samples was carried out in hot $80 \%$ ethanol. After 5 minute of sample centrifugation at $5000 \mathrm{rpm}$, the residue was obtained. Addition of 6.5 $\mathrm{ml}$ of $52 \%$ perchloric acid and $5 \mathrm{ml}$ of distilled water into residue was performed. Centrifugation of solution and supernatant separation were carried out. The supernatant was filtered using a filter paper. The filtration was continued till $100 \mathrm{ml}$ of supernatant was obtained. $1 \mathrm{ml}$ of mixture for a test tube was obtained from addition of $100 \mu 1(0.1$ $\mathrm{ml}$ ) of supernatant to distilled water. Then $4 \mathrm{ml}$ of anthrone reagent were added into the test tube. The mixture was heated for 8 minutes in $100^{\circ} \mathrm{C}$ water bath then cooled at room temperature. Absorbance reading was obtained from spectrophotometer (UV160U) at wavelength $630 \mathrm{~nm}$. The results were reported in $\mathrm{mg}$ glucose per gram dry sample using starch standard curve plotted before [9].

\section{Determination of chlorophyll content}

Leaf samples of $0.5 \mathrm{~g}$ were ground in $10 \mathrm{ml}$ of $80 \%(\mathrm{v} / \mathrm{v})$ acetone using a mortar and pestle. Addition of $0.5 \mathrm{mg}$ calcium carbonate was carried out to avoid the emergence of pheophytin and the filtration of the extract was performed using a filter paper. The samples were washed continuously till they turn colorless. Extract combination, $20 \mathrm{ml}$ was obtained, and the absorbance for chlorophyll (A and B) was recorded at $663 \mathrm{~nm}$ and $645 \mathrm{~nm}$ respectively [10]. The obtained chlorophyll (A and B) was calculated with formulas as below: 
Total chlorophyll: 20.2(A645) + 8.02(A663)

Chlorophyll A: 12.7(A663) - 2.69(A645)

Chlorophyll B: 22.9(A645) - 4.68(A663).

\section{Statistical analysis}

The experiments were conducted in triplicates and the data obtained were analyzed using Microsoft Excel and IBM SPSS Statistics 20.0 software.

\section{Results and Discussion}

\section{Total soluble sugar}

Total soluble sugar content in the leaves of planting materials A-F were determined (Figure 1). The results showed that, except for planting materials B, D and E, there were no significant difference in soluble sugar content detected in other planting materials when supplied with different levels of fertilizer.

In planting material $\mathrm{B}$, soluble sugar produced was $148 \%$ and $150 \%$ higher than $\mathrm{T} 1$ for T2 and T3, respectively. Planting material D had $28 \%$ less soluble sugar in T2 compared to T1. On the other hand, planting material E showed fluctuated level of total soluble sugar where the amount was significantly decreased $47 \%$ compared to control and then increased $50 \%$ from $\mathrm{T} 1$ to $\mathrm{T} 3$. 
A)

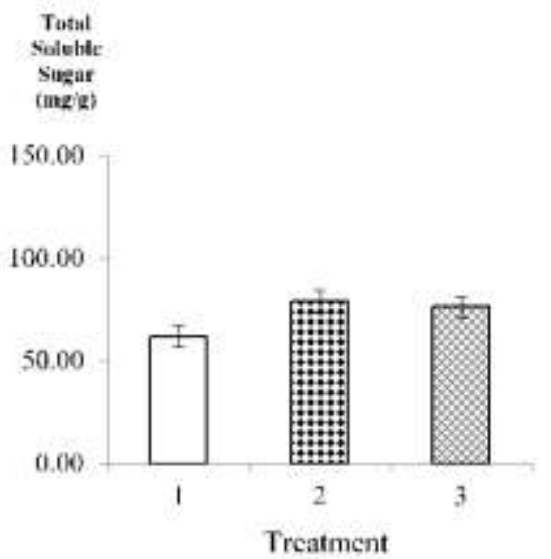

c)

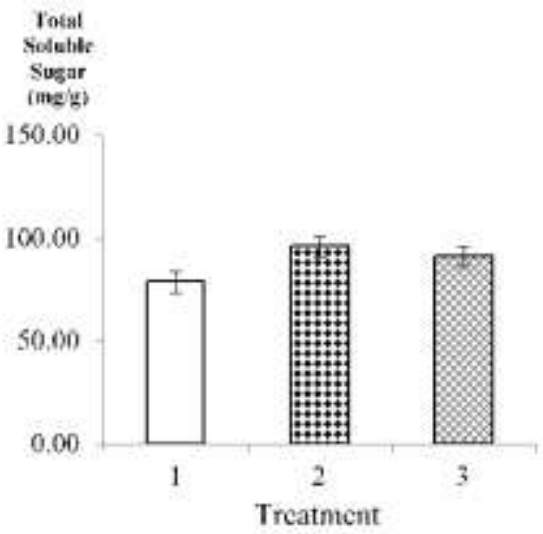

E)

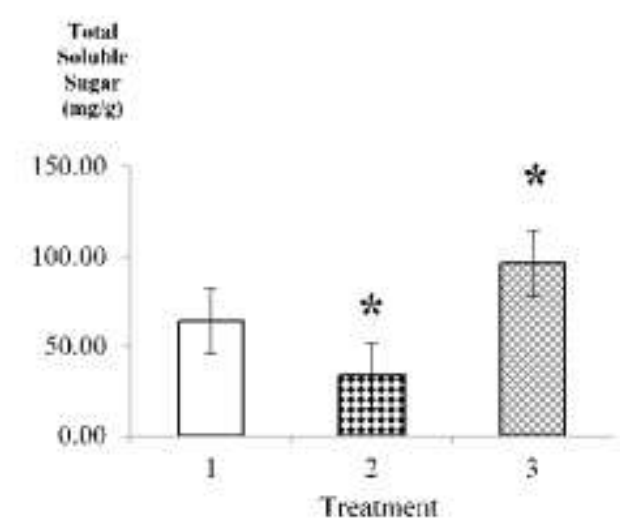

B)

$$
\begin{aligned}
& \text { Tetal } \\
& \text { Nalahte } \\
& \text { Sugar } \\
& \text { (meger }
\end{aligned}
$$

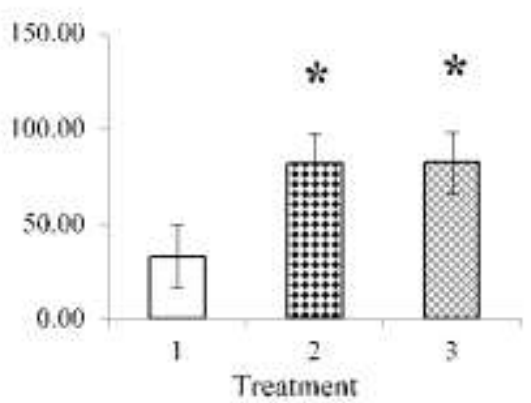

D)
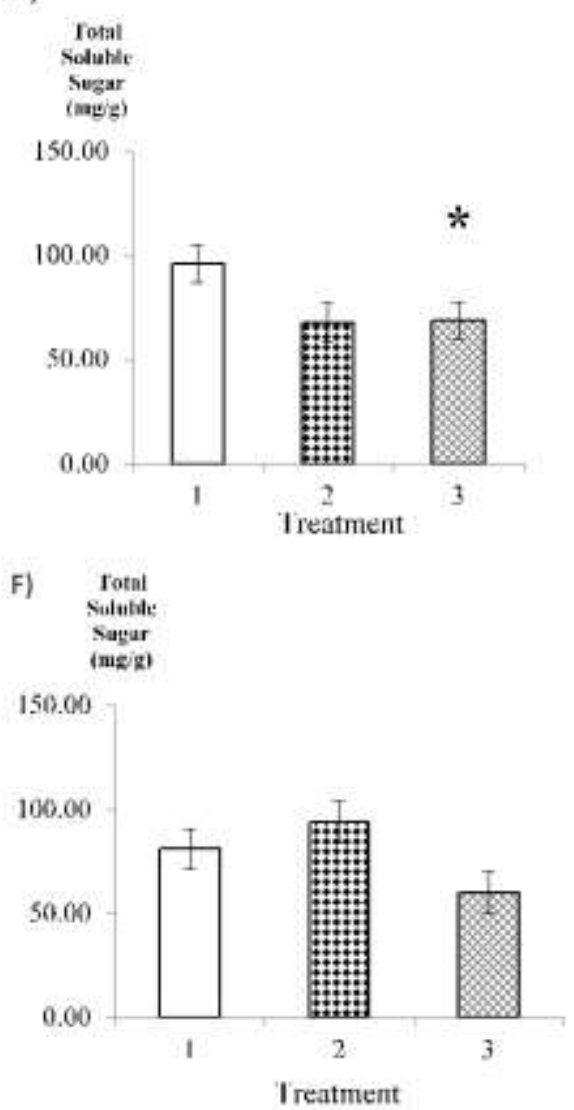

Fig 1 Total soluble sugar content in the leaves of oil palm. (A-F): Planting material A-F treated with different amount of fertilizer. Data are mean \pm standard error, $n=3$. (* indicates significant difference in total soluble sugar content compared to control using t-test; $p<0.05$ )

Soluble sugars supply carbohydrates from source to organs. They have important functions in plants, especially as metabolic resources, cell structural constituents and 
signalling compounds related to plant development. Among the processes are plant flowering, photosynthesis, and other activities which ultimately influence growth and yield production $[11,12,13]$. Photosynthesis will be enhanced by lower level of sugar. Whereas at high level of sugar, plant growth and carbohydrate storage will be promoted [13]. Several genes coding for photosynthetic enzymes were influenced by the levels of soluble sugars in plant cells [14]. Generally, nutrient deficiencies will decrease sugar concentrations, however this is also depends on plant species and other environmental factors. In our study, different planting materials react dynamically towards diferent levels of fertilizer as shown in their soluble sugar production.

\section{Total starch content}

We analyzed the starch content in the leaves of oil palm (Figure 2). As shown in the results, only planting material B had $34 \%$, significantly lower starch content in the leaves of oil palm at T3 compared to T1. Starch content decreased with higher NK fertilizer for this planting material, although the decrease at T2 was not significant. Other planting materials (A,C,D,E and F) supplemented with different levels of NK fertilizer did not show any significant differences. 
A)

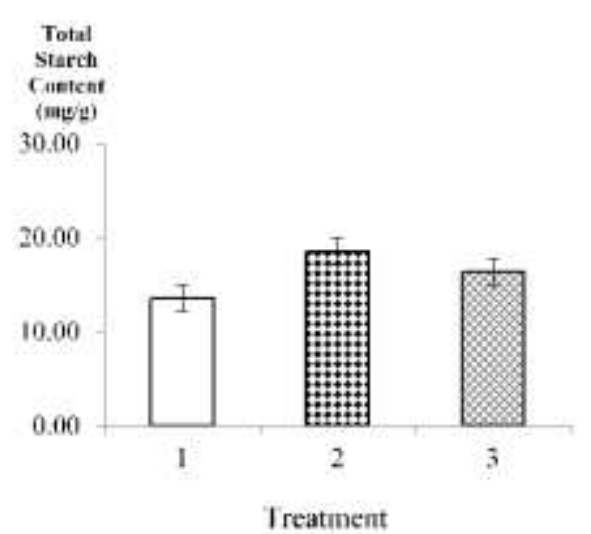

C)

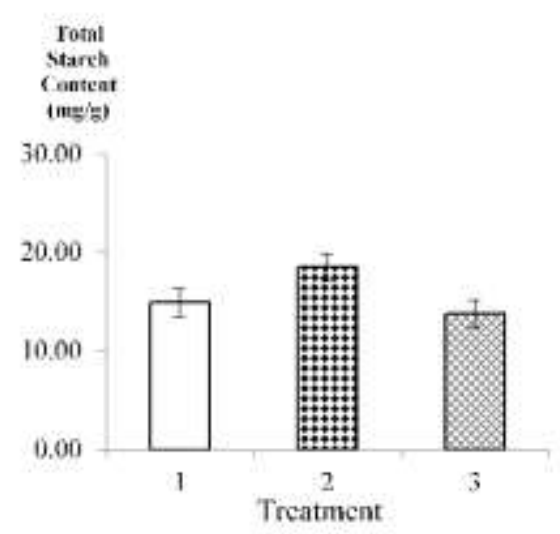

E)

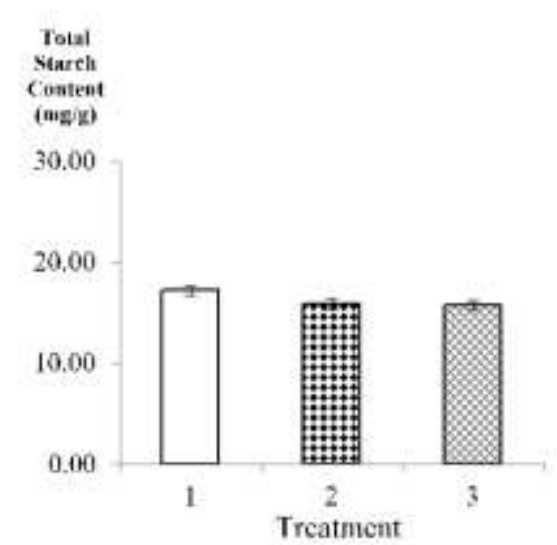

B)

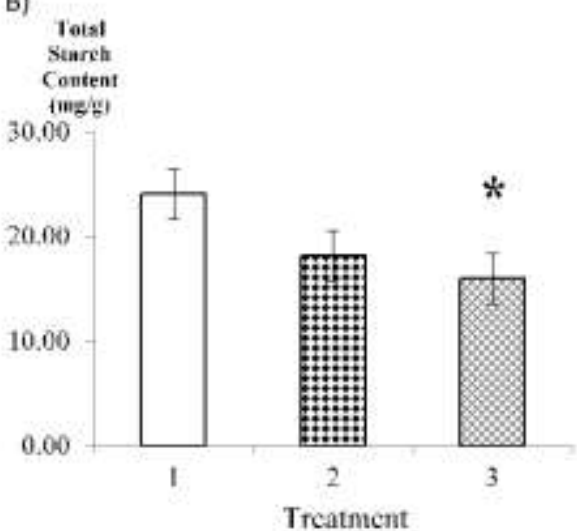

D)

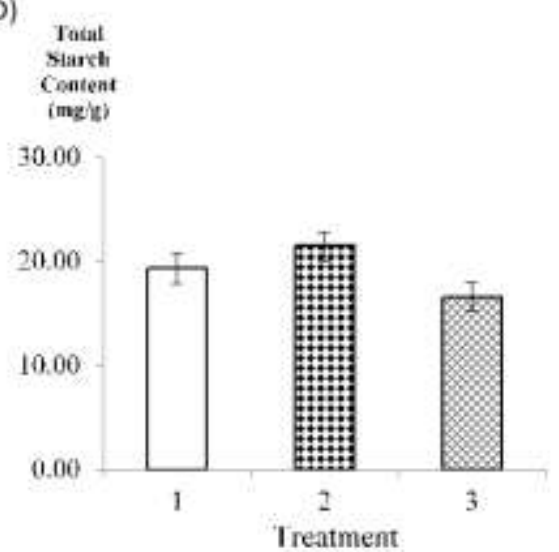

F)

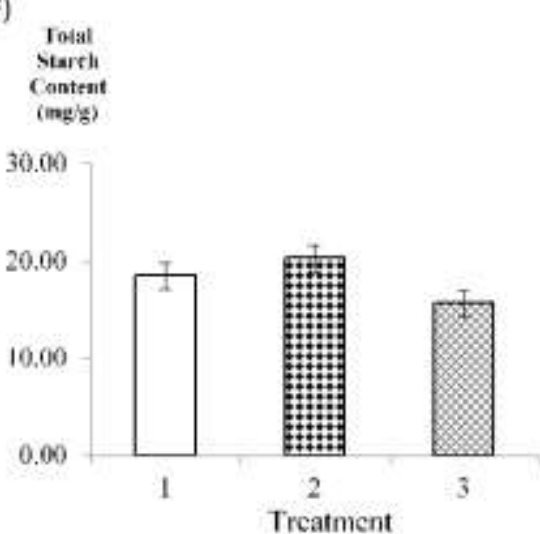

Fig 2 Total starch content in the leaves of oil palm. (A-F): Planting material A-F treated with different amount of fertilizer. Data are mean \pm standard error, $n=3$. (* indicates significant difference in total starch content compared to control using t-test; $p<0.05$ )

Plants store carbohydrate mainly in the form of starch. Hydrolysis of starch will lead to an increase in soluble sugar content [15]. When carbohydrate is mobilized, for instance during pruning, the photosynthetic capacity of oil palm reduces [16]. Inhibition of leaf 
photosynthesis also observed when starch accumulated [17]. We did not found a clear trend on starch content in the leaves of different oil palm planting materials when treated with different levels of fertilizer. A study conducted previously on potato starch and sugar content showed that fertilizer supplied only gave significant effects to starch content at $1 \%$ probability level, but insignificant effect to soluble sugar content [18]. The level of starch and soluble sugar content also influenced by the type of fertilizer applied. Song et al., 2016 reported that organic fertilizer application caused starch accumulation and increased of soluble sugar content in tobacco plants [19].

\section{Total chlorophyll content in oil palm leaves}

Chlorophyll content in plant leaves is another photosynthetic-related parameter that have been determined in this study (Figure 3). The results showed that chlorophyll content in planting material B and D showed significant responses when supplied with slightly higher level of fertilizer. The chlorophyll content dropped significantly at T2 and slightly increased at T3, which were insignificant for both planting materials. The total chlorophyll content for $\mathrm{T} 2$ was $43 \%$ lower than $\mathrm{T} 1$ in planting material $\mathrm{B}$, while the same treatment gave $50 \%$ lower level of chlorophyll content in planting material D when compared to control. 

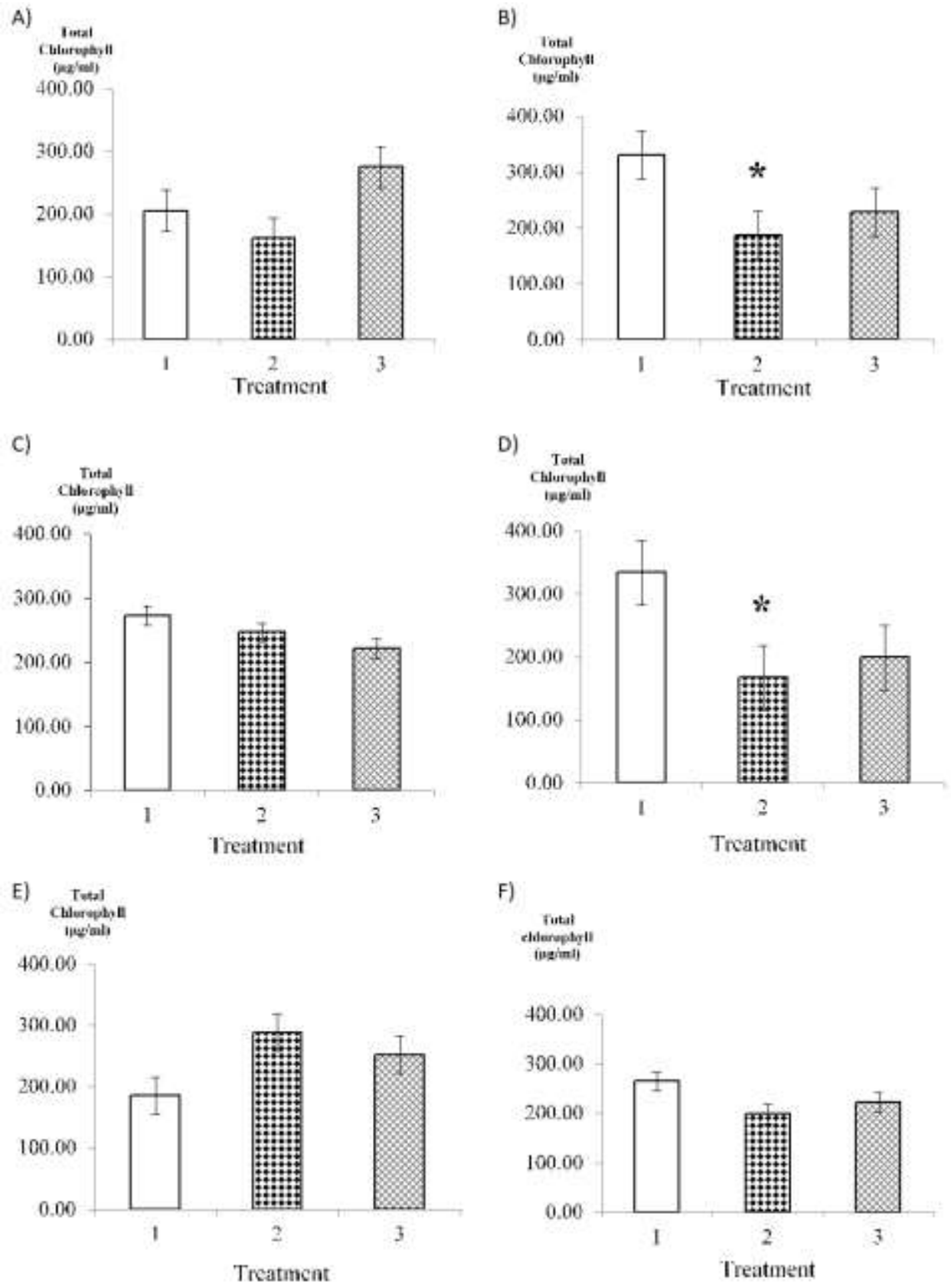

Fig 3 Total chlorophyll content in the leaves of oil palm. (A-F): Planting material A-F treated with different amount of fertilizer. Data are mean \pm standard error, $n=3$. $(*$ indicates significant difference in chlorophyll content compared to control using t-test; $\mathrm{p}<0.05$ )

Chlorophyll content in plants normally influenced by nutrients and light availability besides plant chemical metabolites. Chlorophylls are crucial to plants as they are required for photosynthesis. A study conducted previously on oil palm showed that nitrogen fertilizer did not increase leaf chlorophyll content although the stomatal density increased [20]. 


\section{Conclusion}

Photosynthetic-related properties of oil palm leaves from different planting materials supplemented with different amount of fertilizer were analyzed. Differed responses of each planting material were observed by which no clear trend was recorded in the total soluble sugar, starch content and chlorophyll content in the oil palm leaves. This preliminary findings suggest that further work is required to understand the behaviour of various oil palm planting materials towards chemical fertilizer application.

\section{Acknowledgement}

The authors would like to thank Universiti Teknologi Malaysia for the support of this project from Geran Universiti Penyelidikan (GUP Tier 1) Vote Number: Q.J130000.2545.13H92. We also thank Advance Agriecological Research (AAR) Sdn. Bhd for providing samples and advice related to the project.

\section{Funding}

This study has been funded by Geran Universiti Penyelidikan (GUP Tier 1), Universiti Teknologi Malaysia (UTM): grant number: Q.J130000.2545.13H92.

\section{References}

1. Yew, V. W., et al., The sustainability of oil palm industry in Malaysia: A comprehensive review. Journal of Economic \& Management Perspectives, 2016. 10(4): p.305-310.

2. Begum, H., A.F. Alam, and A. H. Awang, Sustainability of Malaysian oil palm: a critical review. International Journal of Environment and Sustainable Development, 2019. 18(4): p. 409429.

3. Leghari, S.J., et al., Role of nitrogen for plant growth and development: A review. Advances in Environmental Biology, 2016. 10(9): p.209-219.

4. Chaoqiang, J., Z. Chaolong, and W. Huoyan, Effect of nitrogen fertilization on growth and photosynthetic nitrogen use efficiency in Tobacco (Nicotiana tabacum L.). Journal of Life Sciences, 2015. 9(8): p.373-380.

5. Goh, K.J, et al., Fertiliser management in oil palm - Agronomic principles and field practices. Applied Agricultural Research (AAR) Sdn. Bhd, 2018. p.1-44.

6. International Plant Nutrition Institute. Nitrogen Notes 3. Retrieved from: http://www.ipni.net/publication/nitrogenen.nsf/0/FDEE48CFF7600CE585257C13004C7BB0/\$F ILE/NitrogenNotes-EN-03.pdf. Accessed on 1st November 2019.

7. Goh, K. J., Fertilizer recommendation systems for oil palm: Estimating the fertilizer rates. In Proceedings of MOSTA best practices workshops: Agronomy and crop management, 2004. p. 235-268.

8. Prabovo, N. E., et al., Practical use of oil palm nutrient physiological efficiency with regard to nutrient recovery and agronomic efficiencies at different Sumatran sites. 2012. p.1-19.

9. Yemm, E. W. and A.J. Willis, The estimation of carbohydrates in plant extracts by anthrone. The Biochemical Journal, 1954. 57(3): 508-514.

10. Roca, M., K. Chen, and A. Pérez-Gálvez, 6 - chlorophylls, in Handbook on Natural Pigments in Food and Beverages, R. Carle, and R.M. Schweiggert, Editors. 2016, Woodhead Publishing. p.125-158. 
11. Cho, L.H., et al., Roles of sugars in controlling flowering time. Journal of Plant Biology, 2018. 61(3): p.121-130.

12. Pego, J.V., et al., Photosynthesis, sugars and the regulation of gene expression. Journal of Experimental Botany, 2000. 51: p.407-416.

13. Rosa, M., et al., Soluble sugars: Metabolism, sensing and abiotic stress: A complex network in the life of plants. Plant Signaling and Behavior, 2009. 4: p.388-393.

14. Graham, I. A. and T. Martin. Control of photosynthesis, allocation and partitioning by sugar regulated gene expression, in Photosynthesis, Advances in Photosynthesis and Respiration, R.C. Leegood, T.D. Sharkey, and S. Von Caemmerer, 2000. 9: p. 233-248. Springer, Dordrecht.

15. Vaillant-Gaveau, N., et al., Relationships between carbohydrates and reproductive development in Chardonnay grapevine: impact of defoliation and fruit removal treatments during four successive growing seasons. OENO one, 2014. 48(4): p.219-229.

16. Mohd Haniff, H, M. et al., Mobilization of carbohydrates in the oil palm trunk. Poster presented at the PORIM Industry Forum. Hotel Equatorial, Bandar Baru Bangi. 18 December 1997.

17. Ainsworth, E.A., and D.R. Bush, Carbohydrate export from the leaf: a highly regulated process and target to enhance photosynthesis and productivity. Plant Physiology, 2011. 155(1): p. 64-69.

18. Kandi, M. A. S., et al., Effects of different N fertilizer rate on starch percentage, soluble sugar, dry matter, yield and yield components of potato cultivars. Australian Journal of Basic and Applied Sciences, 2011. 5(9): p 1846-1851.

19. Song, Z., et al., Effects of organic fertilizer applications on starch changes in tobacco (Nicotiana tabacum L.) leaves during maturation. Soil Science and Plant Nutrition, 2016. 62(2): p 173-179.

20. Ega, F., Sudradjat and Supijatno, Optimization of nitrogen and phosphorus fertilizer on two years old of oil palm (Elais guineensis Jacq.). Asian Journal of Applied Sciences, 2015. 3(3): p.421-428. 